\title{
Vascular Endothelial Growth Factor and Placenta Growth Factor in Intrauterine Growth-Restricted Fetuses and Neonates
}

\author{
Ariadne Malamitsi-Puchner, Theodora Boutsikou, Emmanuel Economou, \\ Angeliki Sarandakou, Evangelos Makrakis, Dimitrios Hassiakos, \\ and George Creatsas \\ 2nd Department of Obstetrics and Gynecology, University of Athens, 11528 Athens, Greece
}

Received 17 May 2005; accepted 21 June 2005

\begin{abstract}
The angiogenic factors vascular endothelial growth factor (VEGF) and placenta growth factor (PlGF) are respectively up- and downregulated by hypoxia. We aimed to study circulating levels of the above factors in intrauterine growth restriction (IUGR) and to correlate their levels with the customized centiles of the infants. The study included 25 IUGR and 25 appropriate for gestational age (AGA) full-term, singleton infants and their mothers. Maternal (MS), fetal (UC), and neonatal day 1 (N1) and 4 (N4) blood was examined. MS and N1 PlGF, as well as UC VEGF levels correlated with the customized centiles of the infants $(r=0.39, P=.007$, $r=0.34, P=.01$, and $r=-0.41, P=.004$, resp). Furthermore, UC, N1, and N4 VEGF levels were higher in girls $(r=0.36$, $P=.01, r=0.33, P=.02$, and $r=0.41, P=.005$ resp). In conclusion, positive and negative correlations of examined factors with the customized centiles of the infant could rely on placental function and intrauterine oxygen concentrations-both being usually lower in IUGR cases-while higher VEGF levels in girls should possibly be attributed to the stimulating action of estrogens.
\end{abstract}

\section{INTRODUCTION}

Vascular endothelial growth factor (VEGF), a key angiogenic factor in physiological and pathological conditions, exists in five isoforms of 121, 145, 165,189, and 206 amino acids [1]. VEGF exerts its effects by binding with high affinity to two tyrosine kinase receptors, VEGFR1/Flt-1 [2] and VEGFR-2/KDR [3], present on endothelial cells [4]. VEGF has been demonstrated to be a potent stimulator of endothelial cell proliferation [5] and production of the plasminogen activators, required for proteolytic degradation of the extracellular matrix [6]. Both of these actions are markers of angiogenic activity. Particularly, branching angiogenesis mediated by VEGF is due to its binding to both Flt- 1 and KDR receptors. The expression of VEGF and KDR is more pronounced during early gestation, particularly the first two trimesters, and decline with progression of pregnancy [7].

Placenta growth factor (PlGF) belongs to the same family and shares with VEGF 53\% homology [8]. It has been shown to be a very weak stimulator of endothelial

Correspondence and reprint requests to Ariadne MalamitsiPuchner, Neonatal Division, 2nd Department of Obstetrics and Gynecology, University of Athens, 11528 Athens, Greece; malamitsi@aias.gr; amalpu@aretaieio.uoa.gr cell chemotaxis and proliferation at physiological concentrations $[9,10]$. On the other hand, PlGF potentiates the action of low doses of VEGF on microvascular endothelial cells [11]. PlGF binds Flt-1 but not KDR [11] and results in nonbranching angiogenesis $[12,13]$. PlGF and Flt-1 expression increases in the last trimester, towards term [14].

Oxygen is thought to be a major regulator of the balance between VEGF and PlGF function [15]. PlGF expression is stimulated under elevated $\mathrm{PO}_{2}$ and downregulated by a low $\mathrm{PO}_{2}[15,16]$, whereas VEGF and its receptors are upregulated by a low $\mathrm{PO}_{2}$ [17].

Intrauterine growth restriction (IUGR) is commonly associated with an altered placental angiogenesis, due to impaired placental oxygenation [18]. In addition, IUGR is a significant cause of infant mortality and morbidity [19]. The majority of IUGR cases present an asymmetric pattern of growth, usually associated with abnormalities in placental structure and function. The latter are responsible for deprivation of the developing fetus of sufficient oxygen and nutrients, required for his or her optimal growth [20].

In this study, we hypothesized that circulating levels of VEGF and PlGF in IUGR fetuses and neonates should differ from respective levels found in appropriate for gestational age (AGA) fetuses and neonates, as the former suffer from in utero hypoxia and present restricted growth and development. Therefore, we aimed to determine and 
TABLE 1. Demographic data of participating infants (intrauterine growth-restricted (IUGR), or appropriate for gestational age (AGA)) and of their mothers.

\begin{tabular}{lcr}
\hline & IUGR $n=25$ & AGA $n=25$ \\
\hline Maternal age (years) & $30.9 \pm 5.5^{*}$ & $28.9 \pm 3.7^{*}$ \\
Gestational age (weeks) & $38.2 \pm 1.7^{*}$ & $38.9 \pm 1.8^{*}$ \\
Birth weight (g) & $2273 \pm 258.7^{*}$ & $3518 \pm 186.6^{*}$ \\
Gender (male/female) & $12 / 13$ & $14 / 11$ \\
Mode of delivery (VD/ECS) ${ }^{* *}$ & $15 / 10$ & $12 / 13$ \\
\hline$*$ values are mean \pm SD. & & \\
$* *$ VD: vaginal delivery/ ECS: elective cesarean section. & &
\end{tabular}

correlate circulating levels of VEGF and PlGF in IUGR and AGA neonates at time points characteristic for intraand extrauterine life.

\section{MATERIALS AND METHODS}

The study was approved by the Ethics Committee of our teaching hospital and informed consent was acquired from participating mothers.

The study was comprised of 25 IUGR and 25 AGA full-term, singleton infants, born from October 2003 to April 2004, as well as their mothers, all of Greek ethnicity. As IUGR were characterized infants below the 10th customized centile for birth weight. For the calculation of the customized centiles, the principles of the gestation-related optimal weight (GROW) program $[21,22]$ were used. This is a computer-generated antenatal chart that can be customized for each pregnancy, taking into consideration significant determinants of birth weight: gestational age, gender, maternal weight at the beginning of pregnancy, maternal height, ethnic group, and parity. These parameters are entered into the program to adjust the normal birth weight centile limits [21].

The cause of intrauterine growth restriction was identified in each one of our 25 IUGR neonates. The personal, family, and perinatal histories of each parturient were considered and maternal ultrasounds and Doppler studies of the umbilical and middle cerebral artery throughout pregnancy were evaluated. Blood flow studies were in all included cases within normal ranges.

All included in this study IUGR cases were of the asymmetrical pattern. IUGR resulted in 19 cases from hypertension of pregnancy or preeclampsia and in three cases from type I diabetes mellitus. In three further IUGR cases, mothers presented no evident pathological symptoms, but their placentas were very small $(<200 \mathrm{~g})$ and infarcted, despite exclusion of intrauterine infection. However, in all IUGR cases placentas weighed less than $400 \mathrm{~g}$ and were infarcted. Among the 25 mothers, who gave birth to IUGR infants, four reported smoking 5-10 cigarettes per day. In the group of AGA infants, mothers were healthy and three smoked up to 5 cigarettes per day. All placentas were weighed and carefully examined for any sign of malformation. In all IUGR cases placentas were small (less than $400 \mathrm{~g}$ ) and infarcted. Neonates were examined for possible pathological causes leading to restriction, as intrauterine infection or genetic syndromes. Latter cases were excluded from the study. One and five minute Apgar scores were in all IUGR and AGA cases $\geq 7$ and $\geq 8$ respectively.

Demographic data of participating infants, as well as of their mothers are given in Table 1.

Blood was drawn by venipuncture from the mothers (MS) during the first stage of labor or before anesthesia in cases of elective cesarean section, from the doubly clamped umbilical cord (UC-mixed arteriovenous blood), reflecting fetal state, and from the neonates on days 1 (N1) and 4 (N4), characteristic for transition and stabilization to extrauterine life, respectively.

Blood was collected in pyrogen-free tubes and was immediately centrifuged after clotting. The supernatant serum was kept frozen at $-80^{\circ}$ until assay. The determination of VEGF and PlGF was performed by enzyme immunoassay (Quantikine human VEGF, Catalog number DVEOO and Quantikine human PlGF, Catalog number DPGOO, R\&D Systems, Minneapolis, Minn). The minimum detectable concentration, intra-and interassay coefficients of variation were for VEGF $<5 \mathrm{pg} / \mathrm{mL}, 6.7 \%$ and $8.8 \%$ and for $\mathrm{PlGF}<7 \mathrm{pg} / \mathrm{mL}, 7 \%$ and $11.8 \%$ respectively.

For each blood sample drawn, a whole blood count (including platelet count) was also performed.

\section{DATA ANALYSIS}

As not all data presented normal distribution (Kolmogorov-Smirnov test), nonparametric Spearman rank correlation test was applied in the analysis. $P$ values $<.05$ were considered as significant.

\section{RESULTS}

Figures 1 and 2 show the median and ranges of determined parameters in the two groups (IUGR and AGA). Platelet counts were in all cases within normal ranges $\left(\right.$ mean \pm SEM $\times 10^{3} / \mathrm{mL}$; AGA MS: $265 \pm 2.3$, IUGR MS: $273 \pm 3.0$, AGA UC: $312 \pm 3.1$, IUGR UC: $323 \pm 2.8$, AGA $\mathrm{N} 1: 290 \pm 4.8$, IUGR N1: $302 \pm 3.5$, AGA N4: $275 \pm 2.9$, IUGR N4: $273 \pm 2.3$ ).

Statistical significant difference was noted in MS PlGF, being lower in IUGR as compared to AGA cases 


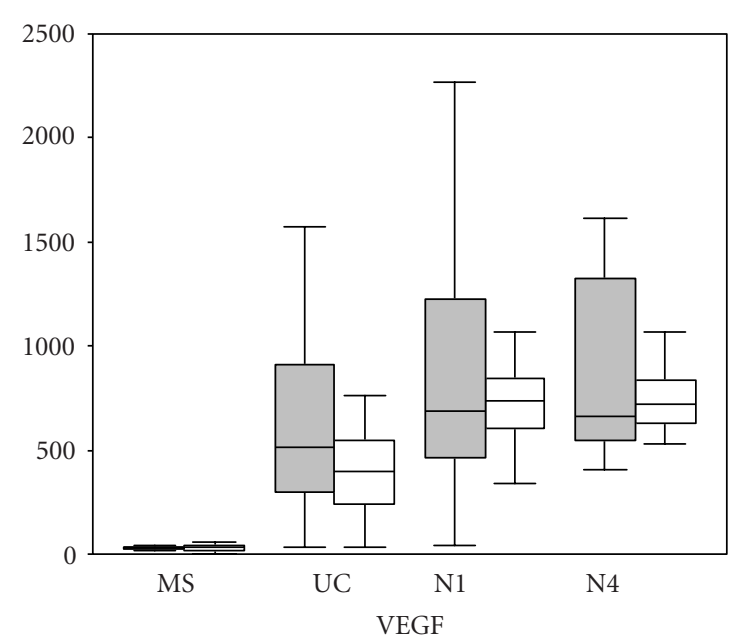

IUGR

AGA

FIgURE 1. Median circulating levels of vascular endothelial growth factor (VEGF (pg/mL)) in the four investigated groups: maternal serum (MS), umbilical cord serum (UC), neonatal day 1 serum (N1), and neonatal day 4 serum (N4) for intrauterine growth-restricted (IUGR) and appropriate for gestational age (AGA) infants.

$(P=.014)$. MS PlGF values were positively correlated with the centile of the infant $(r=0.39, P=.007)$, and his or her birth weight $(r=0.40, P=.006)$. Furthermore, MS PlGF values were negatively correlated with maternal weight at the beginning of pregnancy $(r=-0.30$, $P=.03)$. In addition, UC PlGF values were correlated with neonate's gender, being higher in boys $(r=-0.33$, $P=.01)$, and N1 PlGF values were positively correlated with the centile $(r=0.34, P=.01)$.

On the other hand, MS VEGF values were negatively correlated with maternal weight $(r=-0.36, P=.01)$ and UC VEGF values were negatively correlated with the centile of the infant $(r=-0.41, P=.004)$, and also with the birth weight $(r=-0.30, P=.03)$. Furthermore, UC VEGF values were positively correlated with maternal age $(r=0.32, P=.02)$, and they were higher in girls $(r=0.36, P=.01)$. As for N1 VEGF values, they were higher in girls $(r=0.33, P=.02)$, while N4 VEGF values were positively correlated with maternal age $(r=0.28, P=.04)$ and were also higher in girls $(r=0.41$, $P=.005)$.

\section{DISCUSSION}

To our knowledge, this is the first study to determine VEGF and PlGF circulating levels in IUGR and AGA infants, as well as their mothers.

According to our results, mothers, giving birth to IUGR infants, demonstrate lower PlGF circulating levels than mothers of AGA infants. This finding is in agreement with previous studies, reporting that preeclampsia

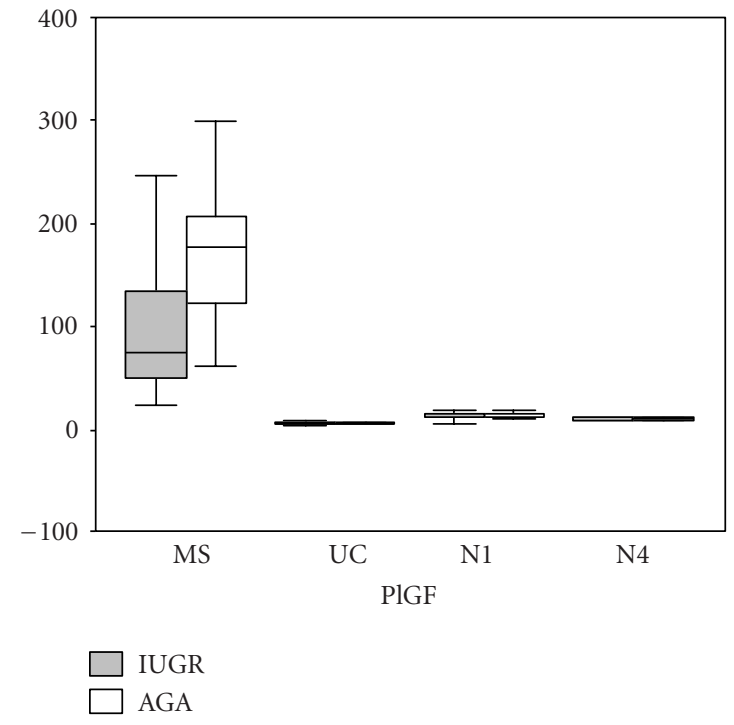

Figure 2. Median circulating levels of placenta growth factor $(\mathrm{PlGF}(\mathrm{pg} / \mathrm{ml}))$ in the four investigated groups: maternal serum (MS), umbilical cord serum (UC), neonatal day 1 serum (N1), and neonatal day 4 serum (N4) for intrauterine growthrestricted (IUGR) and appropriate for gestational age (AGA) infants.

and small-for-gestational-age pregnancies are associated with reduced serum levels of PIGF [23, 24], due to pathologic and smaller placentas [25]. The results of this study extend the existing knowledge by documenting a positive correlation of MS PlGF levels with the centile of the infant, as well as with his or her birth weight.

Several studies advocate that estrogens increase the expression of VEGF in the uterus [26, 27]. Other studies have shown that preeclampsia is characterized by reduced levels of estrogens [28] and thus, in IUGR cases due to preeclampsia, estrogens as well as MS VEGF should be decreased. The negative correlation of MS VEGF and also MS PIGF levels with maternal weight at the beginning of pregnancy might be interpreted on the basis of the higher risk for preeclampsia in obese pregnant women [29].

Studies on circulating VEGF levels in pregnant women provide contradictory results concerning suppression of VEGF [30, 31, 32], presumably by the soluble VEGFR-1 protein, produced and secreted by trophoblasts [33]. In this study, we could demonstrate the presence of circulating VEGF in mothers of both IUGR and AGA infants. However, the origin of the determined VEGF is not clear and could also derive from other sources, such as the ovary [34], and the breast [35]. Nevertheless, MS VEGF levels were not found to correlate with the infant's centile.

The positive correlation of N1 PlGF levels with the centile and respectively the negative correlation of UC VEGF with the centile could be attributed to the chronic in utero action of oxygen, since hypoxia, usually present in IUGR cases, downregulates PlGF and upregulates VEGF in contrast to normoxia $[16,17,18]$. In this study we did 
not consider oxygen concentrations in the blood samples drawn, since these would only represent the current, but not the chronic oxygenation status of the fetal tissues.

The finding of significantly higher UC PlGF levels in boys cannot be explained based on existing literature, while the increased UC, N1, and N4 VEGF levels in girls may rely on stimulated VEGF expression by estrogens [36]. In this respect, the fetal ovary might play a role [37, 38]. However, a previous study of us [32] did not find a prevalence of VEGF in girls, as compared to boys.

Our last finding that UC and N4 VEGF levels were positively correlated with maternal age is to our knowledge reported for the first time. It could be explained taking into consideration that aged mothers are at higher risk for pregnancy complications, among them preeclampsia and IUGR [39] and consequently restricted fetal oxygenation, stimulating VEGF secretion. Nevertheless, the up- or downregulation of VEGF production in IUGR cases is a complicated mechanism, where several factors are implicated.

Lastly, it should be stressed that in this study platelet counts were in all cases within normal ranges and elevated VEGF levels cannot be attributed to elevated platelet counts [40].

In conclusion, according to this study, MS and N1 PlGF, as well as UC VEGF levels correlate with the centile of the infant in positive and negative directions respectively, possibly due to placental function and intrauterine oxygen concentrations, both being usually lower in IUGR cases. Furthermore, UC, N1, and N4 VEGF levels are found higher in girls, possibly due to the stimulating action of estrogens. Further studies are needed to verify above results.

\section{REFERENCES}

[1] Dvorak HF, Brown LF, Detmar M, Dvorak AM. Vascular permeability factor/vascular endothelial growth factor, microvascular hyperpermeability, and angiogenesis. Am J Pathol. 1995;146(5):1029-1039.

[2] de Vries C, Escobedo JA, Ueno H, Houck K, Ferrara $\mathrm{N}$, Williams LT. The fms-like tyrosine kinase, a receptor for vascular endothelial growth factor. Science. 1992;255(5047):989-991.

[3] Terman BI, Dougher-Vermazen M, Carrion ME, Dimitrov D, Gospodarowicz DC, Bohlen P. Identification of the KDR tyrosine kinase as a receptor for vascular endothelial cell growth factor. Biochem Biophys Res Commun. 1992;187(3):1579-1586.

[4] Millauer B, Wizigmann-Voos S, Schnurch H, et al. High affinity VEGF binding and developmental expression suggest Flk-1 as a major regulator of vasculogenesis and angiogenesis. Cell. 1993;72(6): 835846.

[5] Yamane A, Seetharam L, Yamaguchi S, et al. A new communication system between hepatocytes and sinusoidal endothelial cells in liver through vascular endothelial growth factor and Flt tyrosine kinase receptor family (Flt-1 and KDR/Flk-1). Oncogene. 1994;9(9):2683-2690.

[6] Mandriota SJ, Seghezzi G, Vassalli JD, et al. Vascular endothelial growth factor increases urokinase receptor expression in vascular endothelial cells. J Biol Chem. 1995;270(17):9709-9716.

[7] Shiraishi S, Nakagawa K, Kinukawa N, Nakano H, Sueishi K. Immunohistochemical localization of vascular endothelial growth factor in the human placenta. Placenta. 1996;17(2-3):111-121.

[8] Maglione D, Guerriero V, Viglietto G, Delli-Bovi $\mathrm{P}$, Persico MG. Isolation of a human placenta cDNA coding for a protein related to the vascular permeability factor. Proc Natl Acad Sci USA. 1991;88(20):9267-9271.

[9] Hauser S, Weich HA. A heparin-binding form of placenta growth factor (PlGF-2) is expressed in human umbilical vein endothelial cells and in placenta. Growth Factors. 1993;9(4):259-268.

[10] Birkenhager R, Schneppe B, Rockl W, Wilting J, Weich HA, McCarthy JE. Synthesis and physiological activity of heterodimers comprising different splice forms of vascular endothelial growth factor and placenta growth factor. Biochem J. 1996;316(pt 3):703707.

[11] Park JE, Chen HH, Winer J, Houck KA, Ferrara N. Placenta growth factor. Potentiation of vascular endothelial growth factor bioactivity, in vitro and in vivo, and high affinity binding to Flt-1 but not to Flk-1/KDR. J Biol Chem. 1994;269(41):2564625654.

[12] Wilting J, Birkenhager R, Eichmann A, et al. VEGF(121) induces proliferation of vascular endothelial cells and expression of flk-1 without affecting lymphatic vessels of chorioallantoic membrane. Dev Biol. 1996;176(1):76-85.

[13] Kurz H, Wilting J, Sandau K, Christ B. Automated evaluation of angiogenic effects mediated by VEGF and PlGF homo- and heterodimers. Microvasc Res. 1998;55(1):92-102.

[14] Clark DE, Smith SK, Sharkey AM, Charnock-Jones DS. Localization of VEGF and expression of its receptors flt and KDR in human placenta throughout pregnancy. Hum Reprod. 1996;11(5):1090-1098.

[15] Ahmed A, Dunk C, Ahmad S, Khaliq A. Regulation of placental vascular endothelial growth factor (VEGF) and placenta growth factor (PIGF) and soluble Flt-1 by oxygen-a review. Placenta. 2000;21(suppl A):S16-S24.

[16] Khaliq A, Dunk C, Jiang J, et al. Hypoxia downregulates placenta growth factor, whereas fetal growth restriction up-regulates placenta growth factor expression: molecular evidence for "placental hyperoxia" in intrauterine growth restriction. Lab Invest. 1999;79(2):151-170.

[17] Taylor CM, Stevens H, Anthony FW, Wheeler T. Influence of hypoxia on vascular endothelial growth 
factor and chorionic gonadotrophin production in the trophoblast-derived cell lines: JEG, JAr and BeWo. Placenta. 1997;18(5-6):451-458.

[18] Regnault TR, de Vrijer B, Galan HL, et al. The relationship between transplacental $\mathrm{O} 2$ diffusion and placentalexpression of PlGF, VEGF and their receptors in a placental insufficiency model of fetal growth restriction. J Physiol. 2003;550(pt 2):641-656.

[19] Pollack RN, Divon MY. Intrauterine growth retardation: definition, classification, and etiology. Clin $\mathrm{Ob}$ stet Gynecol. 1992;35(1):99-107.

[20] Regnault TR, Galan HL, Parker TA, Anthony RV. Placental development in normal and compromised pregnancies_a review. Placenta. 2002;23(suppl A):S119-S129.

[21] Gardosi J, Chang A, Kalyan B, Sahota D, Symonds EM. Customised antenatal growth charts. Lancet. 1992;339(8788):283-287.

[22] Gardosi J, Mongelli M, Wilcox M, Chang A. An adjustable fetal weight standard. Ultrasound Obstet Gynecol. 1995;6(3):168-174.

[23] Torry DS, Wang HS, Wang TH, Caudle MR, Torry RJ. Preeclampsia is associated with reduced serum levels of placenta growth factor. Am J Obstet Gynecol. 1998;179(6 pt 1):1539-1544.

[24] Tidwell SC, Ho HN, Chiu WH, Torry RJ, Torry DS. Low maternal serum levels of placenta growth factor as an antecedent of clinical preeclampsia. Am J Obstet Gynecol. 2001;184(6):1267-1272.

[25] Taylor RN, Grimwood J, Taylor RS, McMaster MT, Fisher SJ, North RA. Longitudinal serum concentrations of placental growth factor: evidence for abnormal placental angiogenesis in pathologic pregnancies. Am J Obstet Gynecol. 2003;188(1):177-182.

[26] Hyder SM, Huang JC, Nawaz Z, et al. Regulation of vascular endothelial growth factor expression by estrogens and progestins. Environ Health Perspect. 2000;108(suppl 5):785-790.

[27] Albrecht ED, Babischkin JS, Lidor Y, Anderson LD, Udoff LC, Pepe GJ. Effect of estrogen on angiogenesis in co-cultures of human endometrial cells and microvascular endothelial cells. Hum Reprod. 2003;18(10):2039-2047.

[28] Innes KE, Byers TE. Preeclampsia and breast cancer risk. Epidemiology. 1999;10(6):722-732.

[29] O'Brien TE, Ray JG, Chan WS. Maternal body mass index and the risk of preeclampsia: a systematic overview. Epidemiology. 2003;14(3):368-374.

[30] Sharkey AM, Cooper JC, Balmforth JR, et al. Maternal plasma levels of vascular endothelial growth factor in normotensive pregnancies and in pregnancies complicated by pre-eclampsia. Eur J Clin Invest. 1996;26(12):1182-1185.

[31] Lyall F, Greer IA, Boswell F, Fleming R. Suppression of serum vascular endothelial growth factor immunoreactivity in normal pregnancy and in preeclampsia. Br J Obstet Gynecol. 1997;104(2):223228.
[32] Malamitsi-Puchner A, Tziotis J, Protonotariou E, Xyni K, Sarandakou A, Creatsas G. Heparin-binding angiogenic factors (basic fibroblast growth factor and vascular endothelial growth factor) in early neonatal life. Pediatr Res. 1999;45(6):877-880.

[33] Clark DE, Smith SK, He Y, et al. A vascular endothelial growth factor antagonist is produced by the human placenta and released into the maternal circulation. Biol Reprod. 1998;59(6):1540-1548.

[34] Kamat BR, Brown LF, Manseau EJ, Senger DR, Dvorak HF. Expression of vascular permeability factor/vascular endothelial growth factor by human granulosa and theca lutein cells. Role in corpus luteum development. Am J Pathol. 1995;146(1):157165.

[35] Brown LF, Berse B, Jackman RW, et al. Expression of vascular permeability factor (vascular endothelial growth factor) and its receptors in breast cancer. Hum Pathol. 1995;26(1):86-91.

[36] Kang DH, Yu ES, Yoon KI, Johnson R. The impact of gender on progression of renal disease: potential role of estrogen-mediated vascular endothelial growth factor regulation and vascular protection. Am J Pathol. 2004;164(2):679-688.

[37] Pepe GJ, Billiar RB, Leavitt MG, Zachos NC, Gustafsson JA, Albrecht ED. Expression of estrogen receptors $\alpha$ and $\beta$ in the baboon fetal ovary. Biol Reprod. 2002;66(4):1054-1060.

[38] Zachos NC, Billiar RB, Albrecht ED, Pepe GJ. Developmental regulation of baboon fetal ovarian maturation by estrogen. Biol Reprod. 2002;67(4):11481156.

[39] Vanek M, Sheiner E, Levy A, Mazor M. Chronic hypertension and the risk for adverse pregnancy outcome after superimposed pre-eclampsia. Int $J G y$ naecol Obstet. 2004;86(1):7-11.

[40] Banks RE, Forbes MA, Kinsey SE, et al. Release of the angiogenic cytokine vascular endothelial growth factor (VEGF) from platelets: significance for VEGF measurements and cancer biology. $\mathrm{Br} J$ Cancer. 1998;77(6):956-964. 


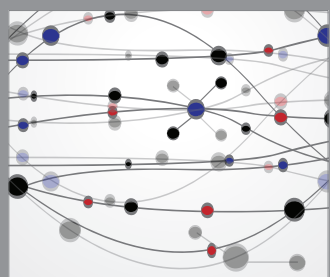

The Scientific World Journal
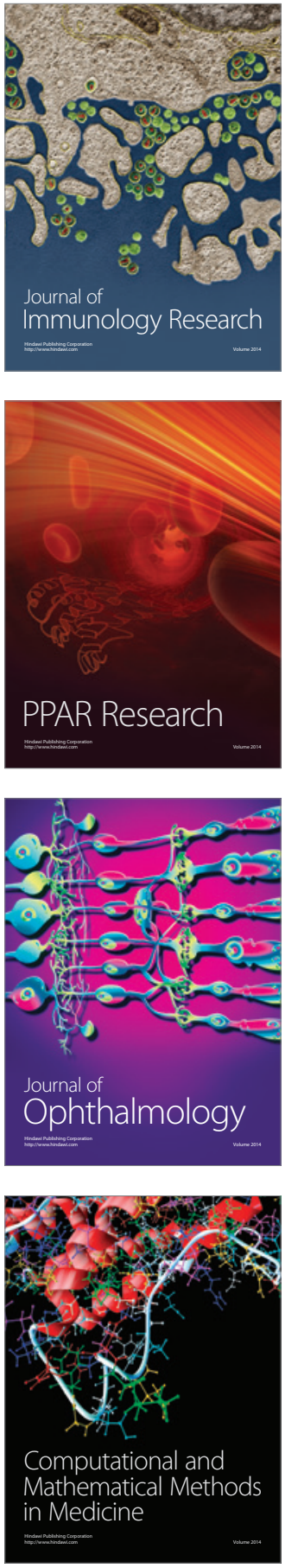

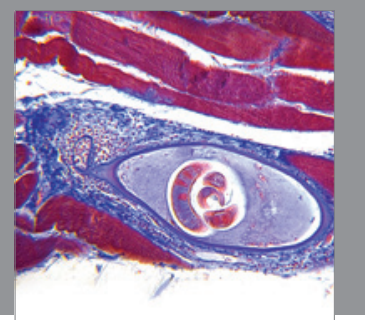

Gastroenterology

Research and Practice
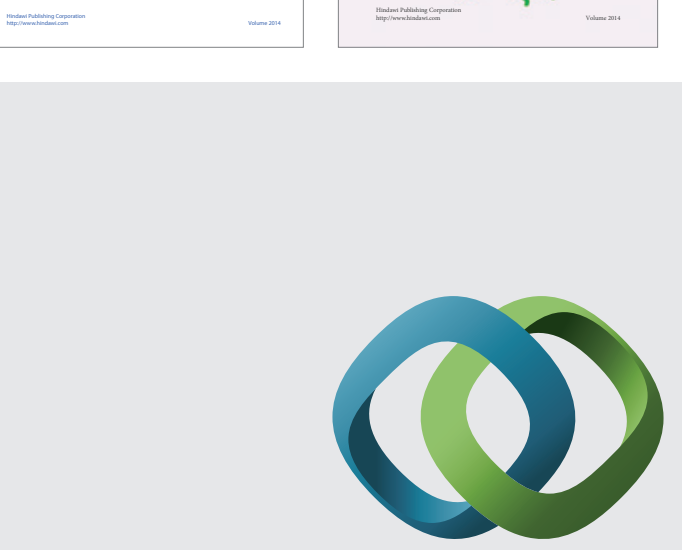

\section{Hindawi}

Submit your manuscripts at

http://www.hindawi.com
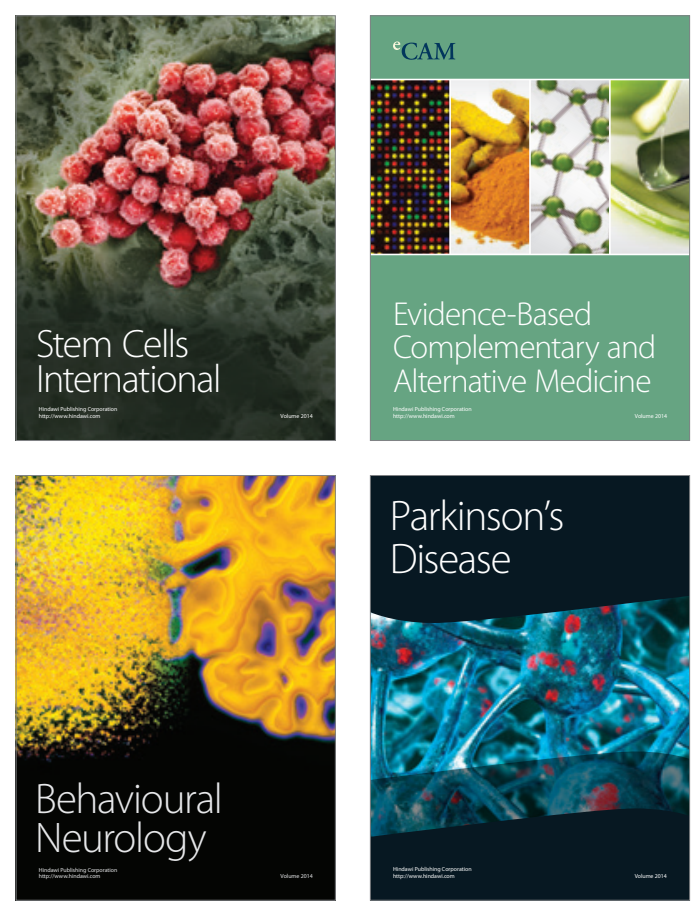

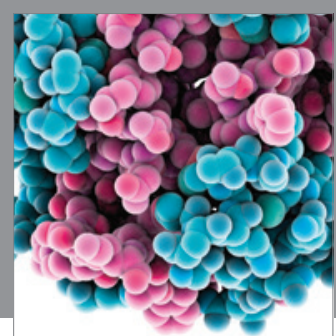

Journal of
Diabetes Research

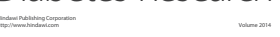

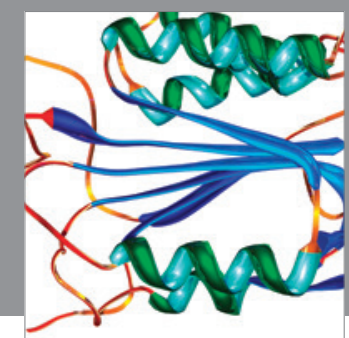

Disease Markers
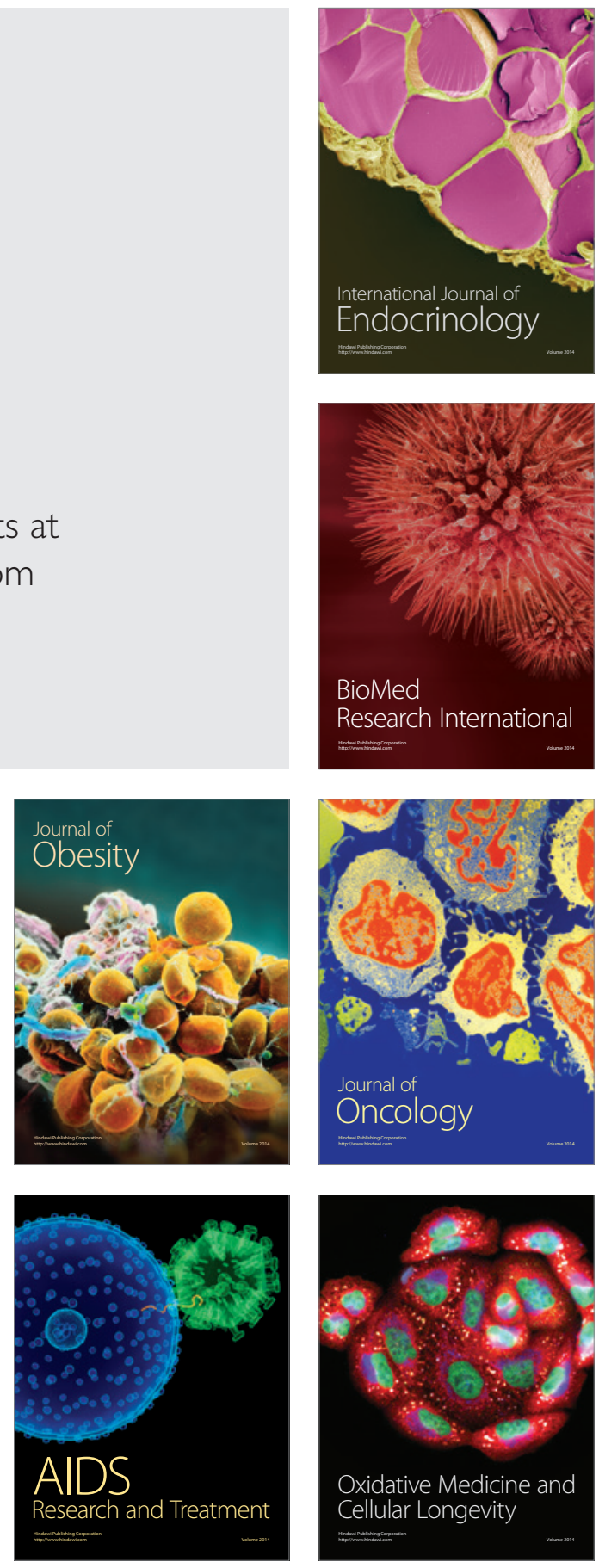